\title{
Accuracy of pulse oximeters in estimating heart rate at rest and during exercise
}

\author{
Y. Iyriboz MD, MPH, S. Powers EdD, PhD, J. Morrow MS, D. Ayers MS and G. Landry MS \\ Exercise Physiology Laboratory, Louisiana State University, Baton Rouge, USA
}

\begin{abstract}
Pulse oximeters are being widely used for non-invasive, simultaneous assessment of haemoglobin oxygen saturation. They are reliable, accurate, relatively inexpensive and portable. Pulse oximeters are often used for estimating heart rate at rest and during exercise. However, at present the data available to validate their use as heart rate monitors are not sufficient. We evaluated the accuracy of two oximeters (Radiometer, ear and finger probe; Ohmeda 3700 , ear probe) in monitoring heart rate during incremental exercise by comparing the pulse oximeters with simultaneous ECG readings. Data were collected on eight men (713 heart rate readings) during graded cycle ergometer and treadmill exercise to volitional fatigue. Analysis by linear regression revealed that general oximeter readings significantly correlated with those of ECG $(r=0.91, P<0.0001)$. However, comparison of heart rate at each level of work showed that oximeter readings significantly $(P<0.05)$ under-estimated rates above 155 beats/min. These results indicate that the use of pulse oximeters as heart rate monitors during strenuous exercise is questionable. This inaccuracy may well originate from the instability of the probes, sweating, other artefacts during exercise, and measurement of different components in the cardiovascular cycle.
\end{abstract}

Keywords: Pulse oximetry, heart rate, exercise

Transcutaneous pulse oximeters are being widely used for non-invasive simultaneous assessment of haemoglobin oxygen saturation ${ }^{1-4}$. Pulse oximeters are often used for estimating heart rate at rest and during exercise. However, at present there are not sufficient data to validate their use as heart rate monitors at rest and during exercise. As accurate measurement of heart rate is becoming increasingly important during exercise, many monitors have become commercially available. The majority of these devices use an infrared source and a transistor photodetector for measuring the pulse ${ }^{5}$. Although these monitors have advantages of time and cost over standard ECG techniques, there have been only a few studies testing their accuracy ${ }^{6,7}$. The purpose of this study was to evaluate the accuracy of two pulse oximeters (Oxi-Radiometer, ear and finger probe (Radiometer America, West Lake, Ohio, USA);

Address for correspondence: Dr Y. Iyriboz, Department of Kinesiology, Louisiana State University, Baton Rouge, Louisiana 70803, USA

(C) 1991 Butterworth-Heinemann Ltd.

0306-3674/91/030162-03
Ohmeda 3700, ear probe (Louisville, Connecticut, USA)) in monitoring heart rate during incremental exercise by comparing them with simultaneous ECG readings.

\section{Method}

Subjects

Eight healthy men volunteered to participate in the study. Their average age was 32.8 years (range 24-49).

\section{Protocol}

The subjects exercised on a cycle ergometer (Monarch 688, Monarch Products Inc, Jacksonville, Texas, USA) and treadmill (Quinton, Uniwork Ergometer 845-644 Programmer, Seattle, Washington, USA) to volitional fatigue. Cycle ergometry began at a work rate of $70 \mathrm{~W}$ and power output was increased by $35 \mathrm{~W}$ at the end of each 2-min stage. Treadmill tests started at $0^{\circ}$ elevation and a speed of $1.7 \mathrm{~m}$.p.h. The speed was increased according to the Bruce protocol ${ }^{8}$ and the grade was elevated by $2.5^{\circ}$ at the end of each $2-\mathrm{min}$ stage.

Toward the end of each stage, during the last $15 \mathrm{~s}$, simultaneous measurements of heart rate were made with direct ECG (Hewlett Packard 1500B, 12-lead, Rockville, Maryland, USA) tracings and readings from the LCD digital displays of the oximeter. Heart rate before the test, at rest and at warm-down (cycle $1 \mathrm{~kg}$ load, 70 r.p.m.; treadmill $0^{\circ}, 1.7 \mathrm{~m}$. p.h.) were also recorded at three consecutive 2-min intervals. Finger and ear lobes were cleaned with $70 \%$ isopropyl alcohol before placing the probes. To minimize motion artefacts, the wiring of ear probes was secured with a head band and the finger probe was taped on the right index finger. Stability of the probes was tested by asking the subjects to move their heads and fingers before the tests started.

\section{Data analysis}

Linear regression analysis was used to determine the predictive capacities of pulse oximeters in estimating heart rate at rest and during exercise. Differences between mean heart rate recorded by oximeters and by ECG were analysed by a paired $t$-test (two-tail). Significance was established at the $P<0.05$ level. 
Table 1. Comparison of heart rate and the correlation between pulse oximeter (POS) and ECG readings

\begin{tabular}{lccc}
\hline Instrument & $\mathrm{n}$ & $\begin{array}{c}\text { Mean heart rate } \\
\text { (s.e.m.) }\end{array}$ & $\begin{array}{c}\text { POS/ECG } \\
\mathrm{r}\end{array}$ \\
\hline Oxi-finger probe & 128 & $120.7(2.5)$ & 0.79 \\
Oxi-ear probe & 201 & $121.0(2.1)$ & 0.95 \\
Ohmeda 3700-ear probe & 201 & $119.5(2.1)$ & 0.92 \\
ECG & 201 & $125.4(2.2)$ & 1.00 \\
\hline
\end{tabular}

All $r$ values significant at $P<0.0001$

Differences between means not significant

\section{Results}

An average of 91.4 heart rate readings per subject was recorded (total 731; treadmill 456, cycle 275). The Oxi-finger probe showed malfunction during the testing of subject No. 6, and was therefore excluded from further evaluation (subjects 7 and 8). Analysis by linear regression revealed that all three oximeter probe readings correlated well with ECG tracings at all work rates (Table 1). A comparison of mean heart rate at all work rates showed that oximeter readings were not significantly different from those of ECG. However, comparison of heart rate at each level of work showed that oximeter readings significantly under-estimated the heart rates ( 16 beats $/ \mathrm{min}$ ) above 155/min (Table 2). Linear regression analysis of heart rate readings below and above $155 / \mathrm{min}$ performed separately showed that previously observed overall significant correlations between ECG and oximeter readings disappeared and became insignificant at rates above $155 / \mathrm{min}$ (Table 3 ).

A scatter diagram plotting pooled oximeter readings against those ECG readings also showed an apparent deviation of oximeter readings at heart rates above 155/min (Figure 1). The heart rate of 155/min corresponds to $89 \%$ of the average maximal rate of our subjects. The average resting rate was $68 / \mathrm{min}$ (range 55-100) and the average maximal heart rate was $174 / \mathrm{min}$ (range 150-190). Plotting of ECG and oximetry readings as a function of maximal heart rate showed that there were no differences between estimates of heart rate between the oximeters and ECG until the subjects reached approximately $89 \%$ of their maximal heart rate. However, above $89 \%$ of the maximal rate (measured by ECG), the oximeter readings significantly under-estimated heart rate at each successive work rate (Figure 2). Data so far studied have been pooled from both treadmill and

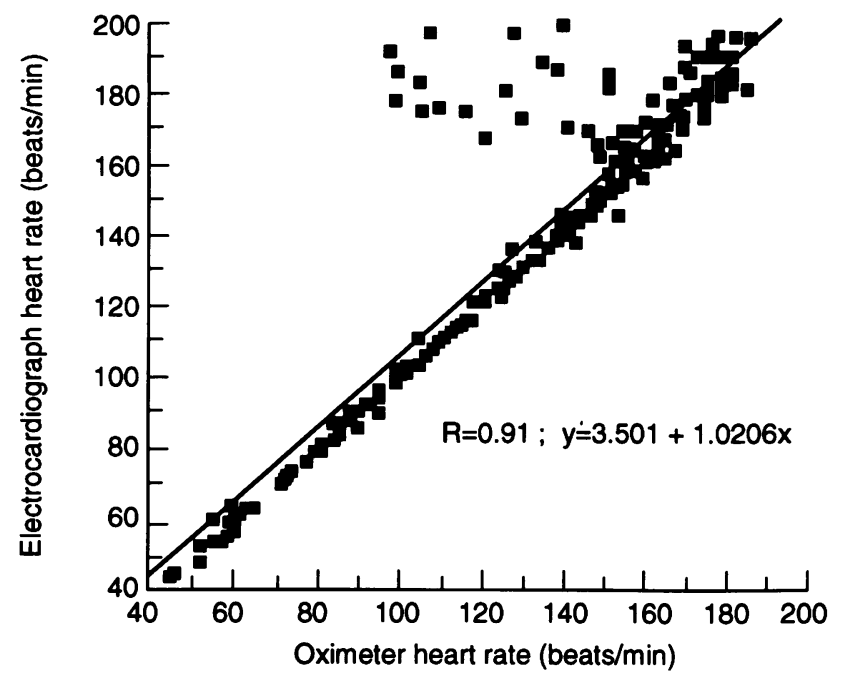

Figure 1. Linear regression line on the scattergram relating the ECG reading of heart rate to the oximeter reading

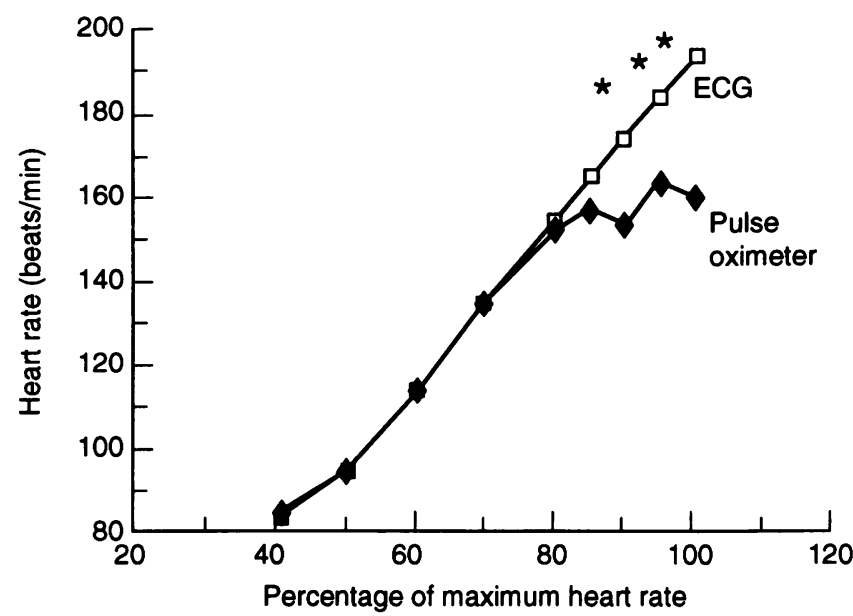

Figure 2. Heart rate (beats/min) with ECG and pulse oximeter readings related to the percentage of maximum heart rate

cycle testing. When the data were analysed separately, as obtained from treadmill and cycle testing, there were no differences from these results.

\section{Discussion}

The results of this study indicate that both pulse oximeters, Oxi with ear and finger probes and Ohmeda 3700 with ear probes, accurately estimate heart rate at rest and during submaximal exercise,

Table 2. Comparison of heart rate below and above 155 beats/min (by ECG) between pulse oximeter (POS) and ECG readings

\begin{tabular}{|c|c|c|c|c|c|}
\hline \multirow[t]{2}{*}{ Instrument } & \multicolumn{2}{|c|}{ ECG rate $<155$ beats/min* } & \multicolumn{2}{|c|}{$E C G$ rate $>155$ beats $/ \mathrm{min}$} & \multirow{2}{*}{$\begin{array}{c}P O S / E C G \\
\mathrm{P}<\end{array}$} \\
\hline & $\mathbf{n}$ & Mean (s.e.m.) & $\mathbf{n}$ & Mean (s.e.m.) & \\
\hline $\begin{array}{l}\text { Oxi-finger probe } \\
\text { Oxi-ear probe } \\
\text { Ohmeda } 3700 \text {-ear probe } \\
\text { ECG }\end{array}$ & $\begin{array}{l}100 \\
161 \\
161 \\
161\end{array}$ & $\begin{array}{l}112.5(2.2) \\
110.8(2.2) \\
112.0(1.9) \\
115.0(1.9)\end{array}$ & $\begin{array}{l}20 \\
28 \\
40 \\
40\end{array}$ & $\begin{array}{l}149.7(4.9) \\
157.3(2.4) \\
154.0(3.4) \\
169.4(1.4)\end{array}$ & $\begin{array}{c}0.0001 \\
0.0001 \\
0.0002 \\
-\end{array}$ \\
\hline
\end{tabular}

* No significant difference between means of oximeters and ECG 
Table 3. Comparison of heart rate data below and above 155 beats/min (by ECG) obtained by pulse oximeter with ECG readings (linear regression)

\begin{tabular}{|c|c|c|c|c|c|c|}
\hline \multirow[t]{2}{*}{ Instrument } & \multicolumn{3}{|c|}{$\begin{array}{c}\text { ECG rate } \\
<155 \text { beats } / \text { min }\end{array}$} & \multicolumn{3}{|c|}{$\begin{array}{c}\text { ECG rate } \\
>155 \text { beats } / \mathrm{min}\end{array}$} \\
\hline & $\mathbf{n}$ & $r$ & $\mathbf{P}<$ & $\mathbf{n}$ & $r$ & $\mathrm{P}<$ \\
\hline Oxi-finger probe & 100 & 0.80 & 0.001 & 28 & 0.16 & 0.38 \\
\hline Oxi-ear probe & 161 & 0.98 & 0.001 & 40 & 0.16 & 0.38 \\
\hline Ohmeda ear probe & 161 & 0.96 & 0.001 & 40 & 0.17 & 0.30 \\
\hline
\end{tabular}

both on the treadmill and the cycle ergometer, below $89 \%$ of maximal heart rate. However, at work rates above $89 \%$, both oximeters tend to under-estimate heart rate by $9 \%$ ( 16 beats $/ \mathrm{min})$. Similar findings have been reported by other studies ${ }^{6,9}$. It is possible that during heavy exercise with excessive movement of the ear lobes, fingers and the probes, oscillations of the pulse presure wave form are distorted in a way which interferes with either transmissions from the infrared source or reception by the photodetector. At this time, this seems to be the only possible explanation, since we have observed a high and significant correlation between the readings when the heart rate is below 155 beats $/ \mathrm{min}$.

It is possible to try to explain the inaccuracy by attributing it either to comparison of two different methods, each measuring a different component of the cardiovascular cycle, or a difference in the time sequence of measurements. The digital displays presented by the pulse oximeters are a rolling average taken over a fixed time period. In the case of Ohmeda, this time period is $12 \mathrm{~s}$ for slow mode and $6 \mathrm{~s}$ for fast mode. However, this will not lead to a discrepancy in comparison of the heart rate measured by ECG with that of the oximeters since ECG heart rate was measured during the last $15 \mathrm{~s}$ of each 2 -min time period, which encompasses one complete slow mode and two complete fast mode time periods of measurement by the pulse oximeter. Measuring the heart rate with the ECG during the last $15 \mathrm{~s}$ of each 2-min period before increasing the workload allows sufficient time for the oximeters to take a rolling average, not only within the last $15 \mathrm{~s}$ but also within the whole time period assigned for each workload which contains ten 12-s periods of slow mode and twenty 6-s periods of fast mode (Figure 3).

\section{Oximeter sequence}

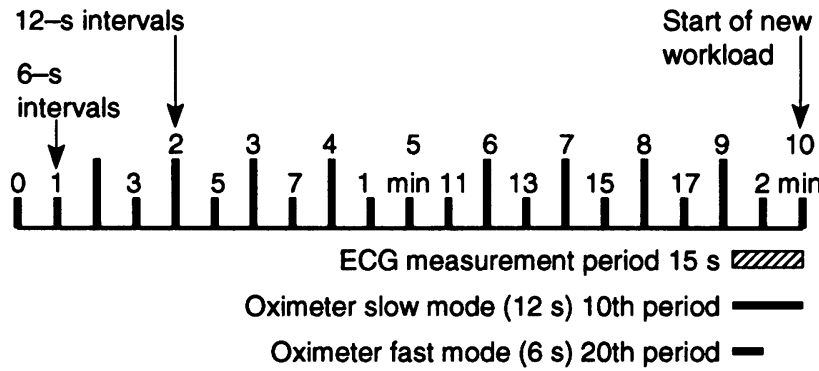

Figure 3. Time sequence of measurements of heart rate by pulse oximeters, with the 10 slow mode (12-s) periods and 20 fast mode (6-s) periods. The ECG measurement is taken only in the final $15 \mathrm{~s}$

In summary, the pulse oximeters studied in this paper accurately estimate heart rate at rest and during submaximal exercise (i.e. at a rate of $<155$ beats/min or $<89 \%$ of maximum), but tend to under-estimate heart rate during heavy exercise. Therefore, at present it is suggested that the use of pulse oximeters to estimate heart rate during exercise should be limited to submaximal work.

\section{References}

1 Jennis MS, Peabody JL. Pulse oximetry: an alternative method for the assessment of oxygenation in newborn infants. Pediatrics 1987; 79: 524-8

2 Maxwell LG, Harris AP, Sendak MJ, Donham RT. Monitoring the resuscitation of preterm infants in the delivery room using pulse oximetry. Clin Pediatr (Phila) 1987; 26: 18-20

3 Ramanathan R, Durand M, Larrazabal C. Pulse oximetry in very low birth weight infants with acute and chronic lung disease. Pediatrics 1987; 79: 612-18

4 Solimano AJ, Smyth JA, Mann TK, Albesheim SG. Pulse oximetry advantages in infants with bronchopulmonary dysplasia. Pediatrics 1987; 78: 844-8

5 Ludwig H. Heart- or respiration-rate calculator. Med Biol Eng Comput 1977; 15: 700-2

6 Burke MJ, Whelan MV. The accuracy and reliability of commercial heart rate monitors. Br J Sports Med 1987; 21: 293-2

7 Leger L, Thivierge M. Heart rate monitors: validity, stability, and functionality. Phy Sports Med 1988; 16: 143-51

8 Bruce RA. Exercise testing for ventricular function. New Engl J Med 1977; 296: 671-5.

9 Landry G, Lawler J, Powers SK. Accuracy of pulse oximetry to estimate heart rate during exercise. South East Chapter of the American College of Sports Medicine Annual Meeting, Jan. 28-30, NC, USA; Winston-Salem, 1988 